CONGENITAL HEART DISEASE

\title{
Evolution of the management approach for pulmonary atresia with intact ventricular septum
}

\author{
Y P Mi, A K T Chau, C S W Chiu, T C Yung, K S Lun, Y F Cheung
}

Heart 2005;91:657-663. doi: 10.1136/hrt.2004.033720

See end of article for authors' affiliations .....................

Correspondence to: Dr Y F Cheung, Division of Paediatric Cardiology, Department of Paediatrics and Adolescent Medicine, The University of Hong Kong, Grantham Hospital, 125, Wong Chuk Hang Road, Aberdeen, Hong Kong, People's Republic of China; xfcheung@hkucc. hku.hk

Accepted 14 August 2004

\begin{abstract}
Objective: To review the evolution of the management approach for pulmonary atresia with intact ventricular septum (PAIVS) in the past two decades and to assess its impact on patient outcomes.

Design and patients: Retrospective review of the management and outcomes of 94 patients (55 male patients) with PAIVS diagnosed between July 1980 and August 2003.

Settings: Tertiary paediatric cardiac centre.

Results: Seven patients died before interventions. Of the remaining 87 patients who underwent intervention at a median age of 9 days (from 1 day to 2 years), 12 had right ventricular oufflow tract reconstruction (RVOTR), 42 had closed pulmonary valvotomy (CPV), and 15 had laser assisted valvotomy with balloon valvoplasty. A systemic-pulmonary shunt was inserted in 18 patients, six of whom had subsequent RVOTR ( $\mathrm{n}=4$ ) or laser assisted valvotomy $(\mathrm{n}=2$ ). Since 1990, catheter intervention accounted for $38 \%$ (17 of 45 ) of the right ventricular oufflow procedures. The mean (SEM) freedom from reintervention was $93(7) \%, 71(12) \%$, and $57(13) \%$ after RVOTR, $75(7) \%$, $40(8) \%$, and $14(6) \%$ after CPV, and $54(13) \%, 24(12) \%$, and $16(10) \%$ affer laser assisted valvotomy at one month, six months, and one year, respectively (RVOTR versus CPV, $\mathrm{p}<0.001$; RVOTR versus laser assisted valvotomy, $p=0.001)$. Low cardiac output syndrome was significantly less common after catheter intervention than after RVOTR (0\% v 44\%, $p=0.003)$ or CPV $(0 \% v 29 \%, p=0.01)$. The overall mean (SEM) survival was $77(5) \%$ and $70(5) \%$ at one and five years, respectively, and the overall mortality was $33 \%$ (29 of 87 ). There were no significant differences in survival between the three groups.

Conclusions: Multiple interventions are often required in the treatment algorithm of PAIVS. The shiff towards increased use of the transcatheter approach has reduced the occurrence of postprocedural low cardiac output syndrome.
\end{abstract}

n pulmonary atresia with intact ventricular septum (PAIVS), morphological diversity of the right ventricle (RV) and coronary arterial anatomy necessitates different management strategies. ${ }^{12}$ Whereas univentricular repair and heart transplantation are options for patients with a diminutive RV or an RV dependent coronary circulation, ${ }^{2}{ }^{3}$ one and a half and biventricular repairs are procedures of choice for those with varying degrees of RV hypoplasia. ${ }^{2-4}$ In the case of RV hypoplasia, early establishment of continuity between the RV and pulmonary trunk is ideal, as it may facilitate a definitive biventricular repair by promoting RV growth. $^{5-8}$

Surgical procedures including RV outflow tract reconstruction (RVOTR $)^{49}$ and pulmonary valvotomy ${ }^{10}{ }^{11}$ have been used to relieve the outflow tract obstruction. In the past decade, transcatheter laser ${ }^{12}$ or radiofrequency assisted ${ }^{13}$ pulmonary valvotomy has been added to the armamentarium for the management of PAIVS. In selected patients with a well developed tripartite RV, the radiofrequency assisted valvotomy procedure has been shown to be more efficacious in decompressing the $\mathrm{RV}$ and associated with lower morbidity than closed surgical valvotomy and BlalockTaussig shunt insertion. ${ }^{14}$

Indeed, recent data from the UK and Ireland collaboration study of pulmonary atresia with intact ventricular septum suggest that increasing numbers of patients are undergoing transcatheter pulmonary valvotomy as the initial procedure. ${ }^{15}$ Nonetheless, the impact of this evolution of management strategy on patient outcomes remains unknown. As alluded to by Daubeney and colleagues, ${ }^{16}$ the rarity of PAIVS limits the population size to $<30$ in more than $90 \%$ of the published series. Given the paucity of clinical data, we reviewed the evolution of management strategies over the past 23 years in 94 infants and children with diagnosed PAIVS and evaluated its impact on patient outcomes.

\section{METHODS}

\section{Patients and data collection}

We reviewed the clinical records of 94 patients (55 male patients) with PAIVS diagnosed between July 1980 and August 2003. The cardiac diagnosis was based primarily on two dimensional and Doppler echocardiography, although angiocardiography had been the mainstay of diagnosis in the earlier years.

The following data were retrieved from the clinical records: demographic data including age, weight, and year at presentation; sex; the initial intervention offered, complications including low cardiac output syndrome and the outcome; the need for and type of subsequent additional interventions; for patients who died, the age at death and cause of death; and for survivors, their further management, follow up duration, and outcomes. Low cardiac output syndrome was defined as the presence of clinical signs and symptoms of the syndrome, as tachycardia, oliguria, cold extremities, or cardiac arrest, with or without a $\geqslant 30 \%$ difference in arterial-mixed venous oxygen saturation or metabolic acidosis. ${ }^{17}$

To determine the morphology of the RV, type of atresia (muscular versus membranous), degree of tricuspid regurgitation, and coronary artery abnormalities, we reviewed all

Abbreviations: CPV, right ventricular outflow tract reconstruction; PAIVS, pulmonary atresia with intact ventricular septum; RV, right ventricular; RVOTR, right ventricular oufflow tract reconstruction 


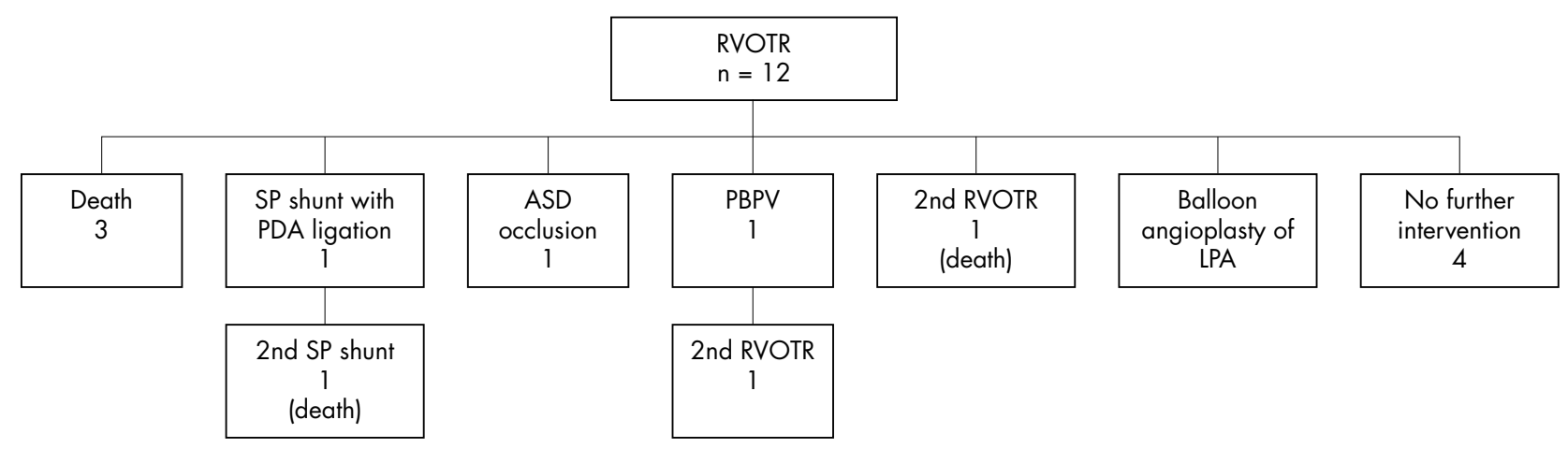

Figure 1 Flowchart showing interventions and outcomes in patients undergoing primary right ventricular outflow tract reconstruction (RVOTR). ASD, atrial septal defect; LPA, left pulmonary artery; PBPV, percutaneous balloon pulmonary valvoplasty; PDA, patent ductal artery; SP, systemic-pulmonary.

the echocardiographic and angiographic recordings at presentation. Morphological description of the RV was based on the tripartite classification described by Bull and colleagues and degree of RV hypoplasia was assessed subjectively. Additionally, the tricuspid valve annulus dimension was measured at end diastole in the echocardiographic apical four chamber view and expressed as a z score from normograms based on postmortem data ${ }^{18}$ and contemporary echocardiographic data. ${ }^{19}$ The degree of tricuspid regurgitation was estimated as absent, mild, moderate, or severe either angiographically or echocardiographically. Furthermore, the presence of Ebstein's malformation of the tricuspid valve was noted. The coronary artery anatomy was studied from the RV and aortic root angiograms and the presence of $\mathrm{RV}$ to coronary artery fistulae was noted. An RV dependent coronary circulation was defined by the presence of coronary arterial interruption, stenosis of one or more of the major epicardial arteries, or absent aortocoronary connection.

\section{Surgical and transcatheter interventions}

Ductal patency was maintained by prostaglandin E2 before the surgical or transcatheter procedure. The management strategy for PAIVS has evolved over the past two decades in our institution. Between July 1980 and October 1991, 12 patients underwent RVOTR as the initial procedure. Between December 1984 and December 1996, transventricular closed pulmonary valvotomy (CPV), performed in 42 patients, had become the mainstay of initial management as reported by our group previously. ${ }^{20}$ Since its introduction in January 1996, transcatheter laser assisted valvotomy with balloon pulmonary valvoplasty had been the initial procedure in 15 patients who did not have infundibular atresia and RV dependent coronary circulation. ${ }^{12}$ In patients with severe RV hypoplasia or RV dependent coronary circulation and in those born preterm or having a low birth weight, the initial palliative management was a systemic-pulmonary arterial shunt.

After successful surgical or transcatheter establishment of ventriculopulmonary continuity, prostaglandin infusion was discontinued. If severe systemic oxygen desaturation $(<70 \%)$ occurred, prostaglandin infusion was restarted. Systemic-pulmonary arterial shunt was inserted in patients who remained prostaglandin dependent for three weeks or more and earlier in those who developed severe hypoxaemia despite prostaglandin infusion. On the other hand, if the patient showed evidence of progressive heart failure, ductal ligation was performed.

\section{Follow up}

All of the survivors had serial echocardiographic assessment to monitor RV growth, severity of residual RV outflow obstruction, and degree of tricuspid and pulmonary regurgitation. The severity of pulmonary regurgitation was graded as the ratio of the colour jet diameter to pulmonary annulus in the parasternal short axis at the level of the RV outflow tract. ${ }^{21}$ For patients with significant RV outflow obstruction (a systolic pressure gradient $>50 \mathrm{~mm} \mathrm{Hg}$ ), balloon valvoplasty or RVOTR was performed as required. For those with persistent systemic oxygen desaturation $(<90 \%)$ despite the absence of significant RV outflow obstruction, device closure of the atrial communication would be performed if a trial of balloon occlusion was tolerated. ${ }^{22}$ A biventricular circulation

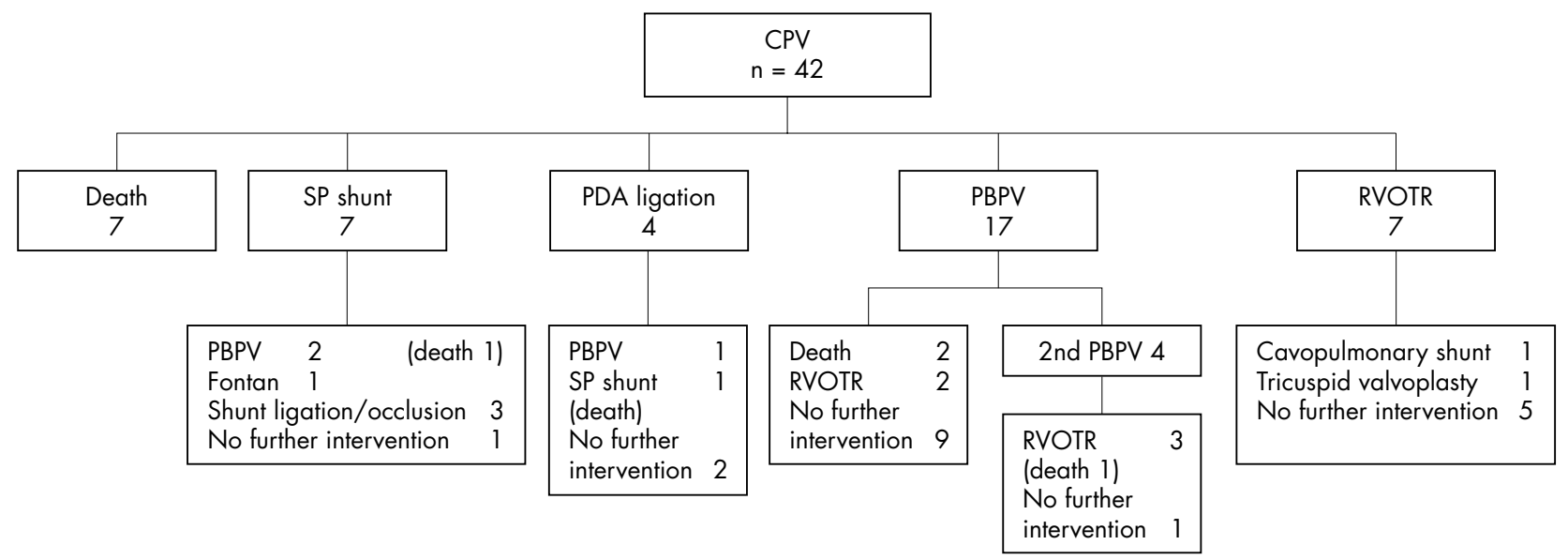

Figure 2 Flowchart showing interventions and outcomes in patients undergoing primary closed pulmonary valvotomy (CPV). 


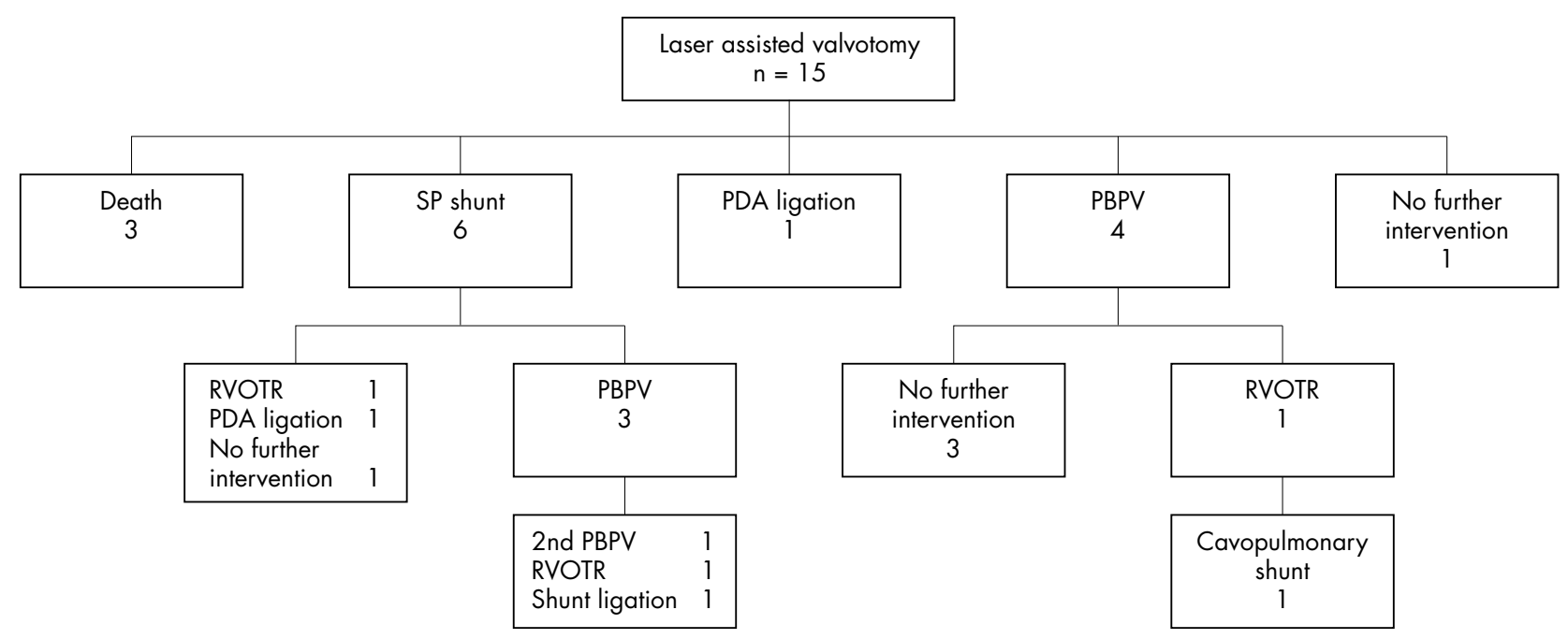

Figure 3 Flowchart showing interventions and outcomes in patients undergoing primary laser assisted valvotomy and balloon valvoplasty.

was considered to have been achieved if there was no systemic oxygen desaturation together with the absence of right to left interatrial shunt as documented by colour flow mapping echocardiographically.

\section{Statistical analysis}

Results are expressed as mean (SD) or median (range) unless otherwise stated. Differences in demographic data, RV morphological variables, and types of initial inventions between survivors and non-survivors were compared by unpaired Student's $t$ test, Mann-Whitney U test, and Fisher's exact test where appropriate. Survival of patients and freedom from the need for multiple interventions were analysed by Kaplan-Meier actuarial survival analysis and groups were compared by log rank test. A probability value of $\mathrm{p}<0.05$ was considered significant. All statistical analyses were performed with SPSS version 10.0 (SPSS, Inc, Chicago, Illinois, USA).

\section{RESULTS}

\section{Patients}

All but two of the 94 patients were ethnic Chinese. The median age at presentation was 1.5 days (range 1 day to 2 years) and the median weight was $3.2 \mathrm{~kg}$ (range 1.4 to 7.2 ).
Three patients had associated syndrome abnormalities, namely Down's syndrome, Noonan's syndrome, and Turner's syndrome. Balloon atrioseptostomy was performed in 15 patients. The atresia was membranous in 88 (93.6\%) and muscular in six $(6.4 \%)$ patients. Four $(4.3 \%)$ patients had Ebstein's malformation of the tricuspid valve. Although $20(21.3 \%)$ had RV to coronary artery fistulae, only one (1.1\%) patient had RV dependent coronary circulation diagnosed.

\section{Surgical and transcatheter management}

Seven patients died before any possible interventions. Of the remaining 87 patients who underwent interventions at a median age of 9 days (range 1 day to 2 years), 12 (14\%) had RVOTR, 42 (48\%) had CPV, 15 (17\%) had laser assisted pulmonary valvotomy with balloon valvoplasty, and 18 (21\%) had insertion of a modified Blalock-Taussig shunt as the initial intervention. Of the 18 patients with shunt insertion, six eventually had their RV outflow opened by either RVOTR $(\mathrm{n}=4)$ or laser assisted valvotomy $(\mathrm{n}=2)$. Figures 1 to 4 summarise the various interventions performed and the outcomes.

Before 1990, the RV outflow was rendered patent by surgery, RVOTR in 10 and CPV in 20 patients. On the other hand, of the 45 patients undergoing RV outflow procedures

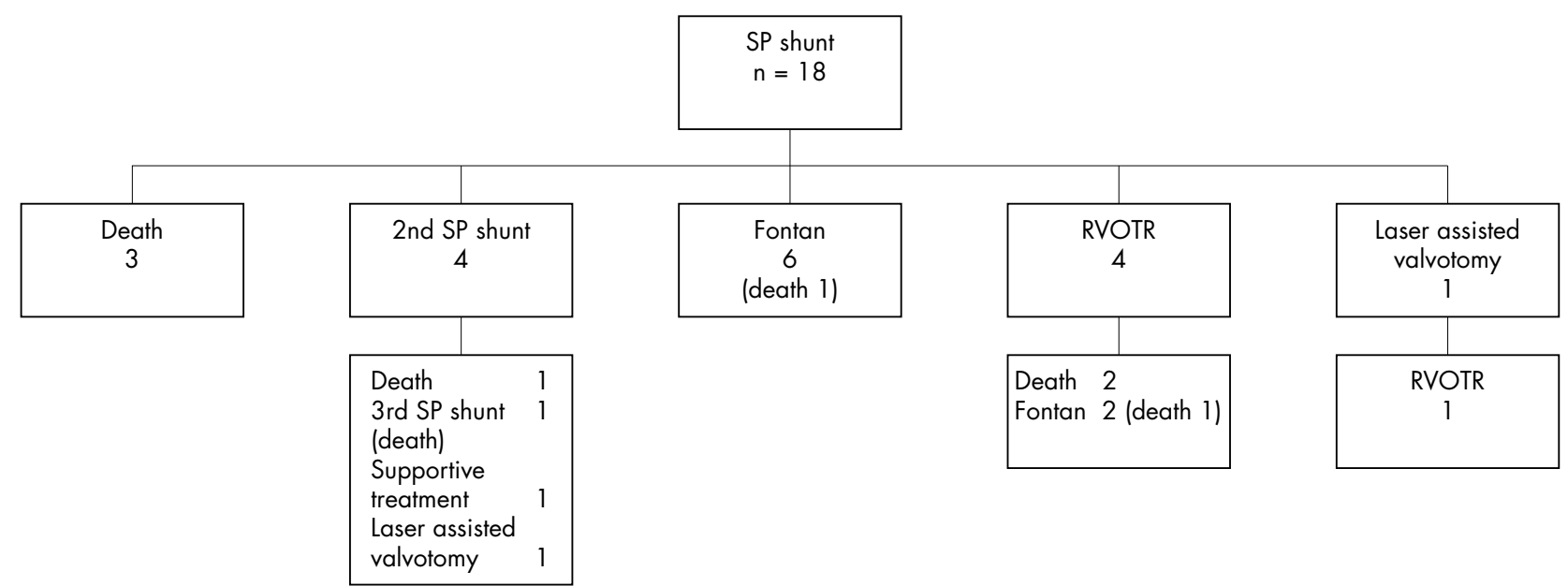

Figure 4 Flowchart showing interventions and outcomes in patients undergoing primary systemic-pulmonary (SP) shunt. 


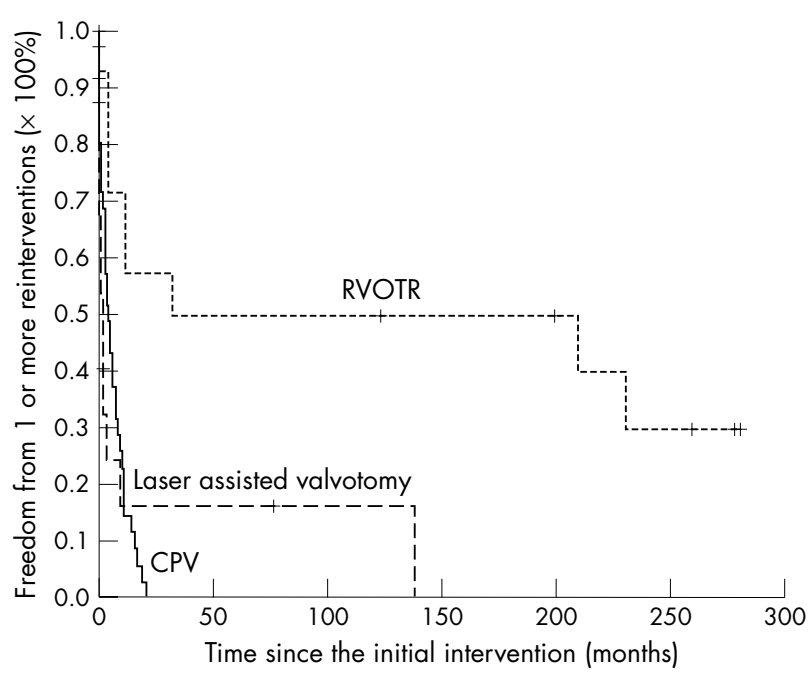

Figure 5 Freedom from $\geqslant 1$ interventions after primary surgical or catheter opening of the right ventricular oufflow tract.

after 1990, 17 (38\%) had transcatheter interventions, 22 had CPV, and six had RVOTR $(\mathrm{p}<0.001)$.

Regardless of the initial intervention for establishment of RV-pulmonary continuity, the need for one or more additional interventions was common. Overall, 52 of the 75 (69\%) patients who had their RV outflow opened required additional interventions. The second interventions were balloon pulmonary valvoplasty in 22 patients, systemicpulmonary shunt insertion with $(\mathrm{n}=6)$ or without ligation $(\mathrm{n}=8)$ of arterial duct in 14, RVOTR in nine, isolated ligation of arterial duct in five, device occlusion of atrial septal defect in one, and balloon angioplasty for left pulmonary artery stenosis in one. Twenty four patients required three interventions and eight patients required more than three interventions.

The mean (SEM) freedom from reintervention was 93 (7)\%, 71 (12)\%, and 57 (13)\% after RVOTR, 75 (7)\%, $40(8) \%$, and $14(6) \%$ after CPV, and $54(13) \%, 24(12) \%$, and $16(10) \%$ after laser assisted valvotomy at one month, six months, and one year, respectively (fig 5). The need for reintervention was

Table 1 Causes of death after surgical and transcatheter interventions

\begin{tabular}{|c|c|}
\hline Cause of death & $\begin{array}{l}\text { Number of } \\
\text { patients }\end{array}$ \\
\hline \multicolumn{2}{|l|}{ RVOTR $(n=8)$} \\
\hline Low cardiac output syndrome & 7 \\
\hline Sudden death of unknown cause & 1 \\
\hline \multicolumn{2}{|l|}{$\operatorname{CPV}(n=10)$} \\
\hline Low cardiac output syndrome & 7 \\
\hline Subcapsular haematoma of liver & 1 \\
\hline Sudden death of unknown cause & 2 \\
\hline \multicolumn{2}{|l|}{ Laser assisted valvotomy $(\mathrm{n}=3$ ) } \\
\hline Perforation of RVOT & 1 \\
\hline Sepsis & 1 \\
\hline Sudden death of unknown cause & $i$ \\
\hline \multicolumn{2}{|l|}{ SP shunt $(n=6)$} \\
\hline Uncontrolled gastrointestinal bleeding & 1 \\
\hline Necrotising enterocolitis & 1 \\
\hline Sudden death of unknown cause & 4 \\
\hline \multicolumn{2}{|l|}{ PBPV $(n=1)$} \\
\hline Catheter induced arrhythmia & 1 \\
\hline Post-Fontan procedure $(n=1)$ & \\
\hline Low cardiac output syndrome & 1 \\
\hline \multicolumn{2}{|c|}{$\begin{array}{l}\text { CPV, closed pulmonary valvotomy; PBPV, percutaneous balloon } \\
\text { pulmonary valvoplasty; RVOTR, right ventricular oufflow tract } \\
\text { reconstruction; SP, systemic-pulmonary. }\end{array}$} \\
\hline
\end{tabular}

significantly less after RVOTR than after both CPV $(p<0.001)$ and laser assisted valvotomy $(p=0.001)$. However, no significant difference was found between the surgical and laser assisted valvotomy treated patients $(\mathrm{p}=0.88)$.

\section{Complications and mortality}

Low cardiac output syndrome was the main complication after RVOTR, occurring in $44 \%$ (seven of 16) of patients. Of the 42 patients who had CPV, 12 (29\%) developed low cardiac output syndrome, four ( $10 \%)$ required peritoneal dialysis for oliguric renal failure, two $(5 \%)$ had sepsis, and one $(2 \%)$ had the complication of subcapsular haematoma of the liver. Among the 17 patients undergoing catheter interventions, sepsis occurred in one (6\%) and perforation of RV outflow in another $(6 \%)$. Low cardiac output syndrome was hence significantly less common after catheter intervention than after RVOTR ( $p=0.003)$ or CPV $(p=0.01)$.

The overall mortality was $33 \%$ (29 of 87 ). Table 1 summarises the causes of early and late deaths. Early postprocedural mortality (death within one month of intervention) was $31 \%$ (five of 16) after RVOTR, 19\% (eight of 42 ) after CPV, and $12 \%$ (two of 17) after laser assisted valvotomy. The early mortality after palliative shunt operation was $6 \%$ (one of 18). Univariate analyses found no significant differences in demographic data, RV morphological variables, type, and the year of the first intervention between survivors and non-survivors (table 2).

\section{Survival and outcomes}

The overall mean (SEM) survival of the 87 patients undergoing interventions was $85(4) \%, 77(5) \%$, and $70(5) \%$ at one month, one year, and five years, respectively. With regard to individual procedures, the survival was $81(10) \%$, $62(12) \%$, and $56(12) \%$ after RVOTR, $81(6) \%, 76(7) \%$, and $71(7) \%$ after CPV, and $88(8) \%, 82(9) \%$, and $82(9) \%$ after laser assisted valvotomy at one month, one year, and five years, respectively (fig 6). Although survival after primary CPV and laser assisted valvotomy appeared to be better than that after RVOTR, the difference was not significant $(p=0.43$ and $\mathrm{p}=0.22$, respectively).

The 58 survivors were followed up for 11.4 (5.6) years (range 5 months to 23 years). One patient who had CPV and a systemic-pulmonary shunt was lost to follow up after immigration. Of the remaining 57 survivors, 39 (68\%) patients achieved a biventricular circulation, seven (12\%) had a univentricular repair, and two (3.5\%) had a one and a half ventricular repair. Six patients were considered likely candidates for biventricular repair, pending either device occlusion of atrial septal defect or RVOTR. One was considered to be a candidate for one and a half ventricular repair. The remaining two patients were managed conservatively after the initial palliative systemic-pulmonary shunt operation; one of them had hypoplastic pulmonary vasculature and the other had pulmonary hypertension and severe RV hypoplasia.

At the latest follow up, $43(75 \%)$ patients were pink in room air. Of the 39 patients who had completed the biventricular repair, the degree of pulmonary regurgitation was found to be mild in 24, moderate in 13 , and severe in two patients echocardiographically. Procedures pending were device occlusion of atrial septal defect in five, RVOTR in one, and left pulmonary artery stenting in one patient.

\section{DISCUSSION}

The present clinical series from a single institution illustrates the evolution of management strategies over the past two decades for the challenging condition of PAIVS. In patients with a diminutive RV and RV dependent coronary circulation, 
Table 2 Comparisons of demographic, morphological, and surgical data between survivors and non-survivors

\begin{tabular}{|c|c|c|c|}
\hline & Survivors $(n=58$ ) & Non-survivors $(n=29)$ & p Value \\
\hline Age at 1 st procedure (days) & $8(1$ to 307$)$ & $9(1$ to 757$)$ & 0.17 \\
\hline Weight at 1 st procedure $(\mathrm{kg})$ & $3.2(2.0$ to 5.5$)$ & $3.2(2.0$ to 7.2$)$ & 0.66 \\
\hline Sex (male/female) & $33 / 25$ & $17 / 12$ & 1.00 \\
\hline \multicolumn{4}{|l|}{ RV hypoplasia } \\
\hline Mild & 19 & 9 & \multirow[t]{3}{*}{0.37} \\
\hline Moderate & 15 & 5 & \\
\hline Severe & 24 & 15 & \\
\hline \multicolumn{4}{|l|}{ RV morphology } \\
\hline Monopartite & 2 & 2 & \multirow[t]{3}{*}{0.61} \\
\hline Bipartite & 9 & 6 & \\
\hline Tripartite & 47 & 21 & \\
\hline RV-coronary connection (yes/no) & $11 / 47$ & $9 / 20$ & 0.28 \\
\hline \multicolumn{4}{|l|}{ Tricuspid regurgitation } \\
\hline Nil or mild & 17 & 8 & \multirow[t]{3}{*}{0.51} \\
\hline Moderate & 10 & 6 & \\
\hline Severe & 31 & 15 & \\
\hline Ebstein's abnormality (yes/no) & $2 / 56$ & $2 / 27$ & 0.60 \\
\hline \multicolumn{4}{|l|}{ Tricuspid valve dimension } \\
\hline Annulus size $(\mathrm{mm})$ & $10.4(2.9)$ & $9.8(3.4)$ & 0.49 \\
\hline Annulus z score* & $-1.2(1.5)$ & $-1.5(2.4)$ & 0.52 \\
\hline Annulus z score $\dagger$ & $-3.8(2.5)$ & $-3.9(2.7)$ & 0.85 \\
\hline \multicolumn{4}{|l|}{ Type of primary procedure } \\
\hline RVOTR & 7 & 5 & \\
\hline CPV & 30 & 12 & \multirow{3}{*}{0.24} \\
\hline Laser assisted valvotomy & 12 & 3 & \\
\hline SP shunt & 9 & & \\
\hline Year of intervention (before/after 1990) & $26 / 32$ & $9 / 20$ & 0.25 \\
\hline
\end{tabular}

the management is palliative systemic-pulmonary arterial shunt insertion and subsequent univentricular repair. In those with anatomically suitable RVs, laser assisted valvotomy with balloon valvoplasty has become the procedure of choice in our institution. However, regardless of the initial procedure, multiple interventions are often required in the subsequent treatment algorithm, especially for patients after CPV and laser assisted pulmonary valvotomy with balloon dilatation. Nonetheless, the risk of developing low cardiac output syndrome is significantly less after catheter intervention than after either RVOTR or CPV. Although survival of patients after RVOTR appeared worse than survival after CPV or primary catheter intervention, the difference was not significant.

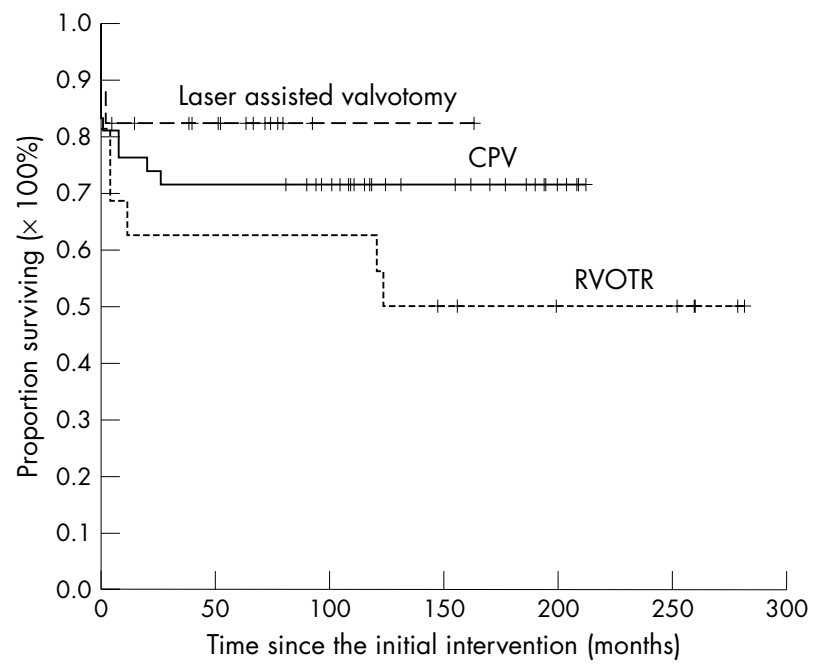

Figure 6 Kaplan-Meier survival estimates for patients after primary surgical and transcatheter interventions for establishment of right ventricular-pulmonary trunk continuity.
Survival of patients with PAIVS has been relatively poor until recently. In the multi-institutional study reported by Hanley and colleagues ${ }^{18}$ that constitutes the largest cohort of surgical patients to date, the overall survival was $64 \%$ at four years. The $56 \%$ and $71 \%$ five year survival of our patients after RVOTR and CPV, respectively, concur with their findings ${ }^{18}$ and those reported previously. ${ }^{11}{ }^{23}$ While transannular patch opening of the RV outflow reduces the chance of residual outflow obstruction, the possibility of acute RV failure, especially in the presence of significant RV hypoplasia (tricuspid valve $\mathrm{z}$ value $<4$ ), remains an issue of concern. ${ }^{18}{ }^{23}$ However, using a selective approach based on the RV growth and coronary artery anatomy, Jahangiri and colleagues ${ }^{9}$ had an excellent surgical result of a zero mortality in 25 patients after RVOTR with or without concomitant systemicpulmonary arterial shunt insertion.

Notwithstanding the significantly improved survival after RVOTR, CPV has its advocates. ${ }^{10} 2022$ Surgical pulmonary valvotomy is a relatively simple procedure that can be performed by the transventricular ${ }^{20}$ or transarterial ${ }^{10}$ route without the need for cardiopulmonary bypass, and it may produce less RV damage and pulmonary regurgitation. ${ }^{22}$ Furthermore, in the presence of RV to coronary communications, it has been suggested that stepwise reduction of RV pressure by initial survival valvotomy and later balloon valvoplasty may potentially avoid compromising myocardial perfusion secondary to RV steal, as the RV pressure usually remains high after the initial valvotomy. ${ }^{10}$ However, as our data show (fig 2), additional procedures are often required after CPV. In the early postoperative period, 19\% (8/42) of patients required a systemic-pulmonary artery shunt after CPV but in the intermediate and long terms, 64\% (27/42) required further relief of residual outflow obstruction by either balloon valvoplasty or RVOTR.

The optimal surgical approach to relieve RV outflow obstruction hence remains controversial. Additionally, the need for concomitant systemic-pulmonary arterial shunt insertion as part of the initial surgery is controversial. 
Concerns have been raised with regard to volume loading of the left ventricle and reduction of systemic diastolic pressure after shunt insertion. ${ }^{3}$ On the other hand, Hanley and colleagues ${ }^{18}$ reported that concomitant shunt insertion during RVOTR improves survival, possibly by preventing significant hypoxia during the early postoperative period.

Data in the literature that compare the outcomes of patients after surgical decompression of the RV with outcomes after transcatheter valvotomy are limited. ${ }^{14}$ In a recent study comparing the results of radiofrequency assisted valvotomy and balloon dilatation with CPV and BlalockTaussig shunt, Alwi and colleagues ${ }^{14}$ concluded that transcatheter intervention is more effective in decompressing the $\mathrm{RV}$ and is associated with a lower morbidity. It is important to note, however, that patients selected for radiofrequency assisted valvotomy have relatively well developed tripartite RVs. The present study, however, failed to confirm a lower reintervention rate after transcatheter opening of the RV outflow. This may be related to our inclusion of patients having moderately hypoplastic RVs (tricuspid valve z score as small as -3.42) for catheter intervention as reported previously. ${ }^{12}$

The atretic pulmonary valve was successfully perforated by laser assistance in 94\% (16 of 17) of our patients, which is comparable with the $75-100 \%$ reported by various investigators. $^{624-27}$ By avoiding primary RV outflow surgery, catheter intervention may reduce the severity of RV damage and morbidity caused by postoperative complications. Nonetheless, perforation of the infundibulum and sepsis are not uncommon complications. ${ }^{64-26}$ At intermediate term follow up, we ${ }^{12}$ and others ${ }^{6}$ have shown that, although the absolute RV and tricuspid annulus dimensions increase after catheter intervention, RV area $^{25}$ and tricuspid annulus ${ }^{625}$ $\mathrm{Z}$ values decrease progressively with somatic growth, suggesting that a normal RV size may not be mandatory for sustaining a biventricular circulation. However, it remains unknown whether the RV growth is similar to that after RVOTR or CPV. Nonetheless, the proportion of patients who have achieved or are likely to achieve a biventricular circulation is similar between patients undergoing primary catheter intervention (11 of $14,79 \%$ ) and those undergoing primary RVOTR or CPV (34 of 43,79\%, p = 1.0). Our data compare favourably with the $64 \%$ ( 16 of 25 ) after catheter intervention as reported by Humpl and colleagues ${ }^{25}$ and the $76 \%$ ( 19 of 25 ) after surgery. ${ }^{22}$

A number of limitations deserve comments. Firstly, the relatively small number of patients undergoing primary catheter intervention might have biased the outcome analysis. Even so, our findings concur with those of a recent study of 30 patients undergoing radiofrequency assisted valvotomy. ${ }^{25}$ Secondly, the management strategies have evolved over a period of two decades. Undoubtedly, the surgical mortality has been reduced significantly in the current era as illustrated by the results of Jahangiri and colleagues. ${ }^{9}$ However, even taking into account the improved postoperative survival data in the present era, there is no suggestion that catheter intervention is a more risky procedure than primary surgical outflow repair.

Despite evolution of management strategy, multiple interventions are still often required in the treatment algorithm of PAIVS. The shift towards primary catheter intervention for patients with PAIVS with a suitable anatomy has reduced the occurrence of postprocedural low cardiac output syndrome. Although the optimal approach to relieve RV outflow obstruction in PAIVS remains controversial, laser assisted valvotomy with balloon valvoplasty proves to be an acceptable alternative. Further studies to compare the cost effectiveness and the severity of pulmonary regurgitation and its impact on long term RV function between surgical and catheter interventions may help to resolve the controversy.

\section{Authors' affiliations}

Y P Mi*, A K T Chau, T C Yung, K S Lun, Y F Cheung, Division of Paediatric Cardiology, Department of Paediatrics and Adolescent Medicine, Grantham Hospital, The University of Hong Kong, Hong Kong, People's Republic of China

C S W Chiu, Division of Cardiothoracic Surgery, Grantham Hospital ${ }^{*}$ Currently working in Children's Hospital of Fudan University, China

\section{REFERENCES}

1 Bull C, de Leval MR, Mercanti C, et al. Pulmonary atresia and intact ventricular septum: a revised classification. Circulation 1982;66:266-72.

2 De Leval M, Bull C, Stark J, et al. Pulmonary atresia with intact ventricular septum: surgical management based on a revised classification. Circulation 1982:66:272-80.

3 Rychik J, Levy H, Gaynor JW, et al. Outcome after operations for pulmonary atresia with intact ventricular septum. J Thorac Cardiovasc Surg 1998;116:924-31

4 Mainwaring RD, Lamberti JJ. Pulmonary atresia with intact ventricular septum: surgical approach based on ventricular size and coronary anatomy. J Thorac Cardiovasc Surg 1993;106:733-8.

5 Patel RG, Freedom RM, Moes CAF, et al. Right ventricular volume determinations in 18 patients with pulmonary atresia and intact ventricular septum: analysis of factors influencing right ventricular growth. Circulation 1980;61:428-40

6 Ovaert C, Qureshi SA, Rosenthal E, et al. Growth of the right ventricle after successful transcatheter pulmonary valvotomy in neonates and infants with pulmonary atresia and intact ventricular septum. J Thorac Cardiovasc Surg 1998:115:1055-62.

7 Hanseus K, Biorkhem G, Lundstrom NR, et al. Cross-sectional echocardiographic measurements of right ventricular size and growth in patients with pulmonary atresia and intact ventricular septum. Pediatr Cardiol 1991;12:135-42.

8 Giglia TM, Jenkins KJ, Matitiau A, et al. Influence of right heart size on outcome in pulmonary atresia with intact ventricular septum. Circulation 1993;88:2248-56.

9 Jahangiri $M$, Zurakowski D, Bichell D, et al. Improved results with selective management in pulmonary atresia with intact ventricular septum. J Thorac Cardiovasc Surg 1999;118:1046-55.

10 Sano S, Ishino K, Kawada M, et al. Staged biventricular repair of pulmonary atresia or stenosis with intact ventricular septum. Ann Thorac Surg 2000;70:1501-6

11 Bull C, Kostelka M, Sorensen K, et al. Outcome measures for the neonatal management of pulmonary atresia with intact ventricular septum. J Thorac Cardiovasc Surg 1994; 107:359-66.

12 Cheung YF, Leung MP, Chau AKT. Usefulness of laser-assisted valvotomy with balloon valvoplasty for pulmonary valve atresia with intact ventricular septum. Am J Cardiol 2002;90:438-42.

13 Wang JK, Wu MH, Chang Cl, et al. Outcomes of transcatheter valvotomy in patients with pulmonary atresia and intact ventricular septum. Am J Cardiol 1999;84: 1055-60.

14 Alwi M, Geetha K, Bilkis AA, et al. Pulmonary atresia with intact ventricular septum percutaneous radiofrequency-assisted valvotomy and balloon dilation versus surgical valvotomy and Blalock-Taussig shunt. J Am Coll Cardiol 2000;35:468-76

15 Fricker FJ, Zuberbuhler JR. Pulmonary atresia with intact ventricular septum. In: Anderson RH, Baker EJ, Macartney FJ, eds. Pediatric cardiology. Edinburgh: Churchill Livingstone, 2002:1187.

16 Daubeney PEF, Delany DJ, Anderson RH, et al. Pulmonary atresia with intact ventricular septum: range of morphology in a population-based study. J Am Coll Cardiol 2002;39:1670-9.

17 Hoffman TM, Wernovsky G, Atz AM, et al. Prophylactic intravenous use of milrinone after cardiac operation in pediatrics (PRIMACORP) study. Am Heart J 2002;143:15-21

18 Hanley FL, Sade RM, Blackstone EH, et al. Outcomes in neonatal pulmonary atresia with intact ventricular septum: a multiinstitutional study. J Thorac Cardiovasc Surg 1993;105:406-27.

19 Daubeney PEF, Blackstone EH, Weintraub RG, et al. Relationship of the dimension of cardiac structures to body size: an echocardiographic study in normal infants and children. Cardiol Young 1999;9:402-10.

20 Leung MP, Lo RNS, Cheung $\mathrm{H}$, et al. Balloon valvuloplasty after pulmonary valvotomy for babies with pulmonary atresia and intact ventricular septum. Ann Thorac Surg 1992;53:864-70.

21 Williams RV, Minich LL, Shaddy RE, et al. Comparison of Doppler echocardiography with angiography for determining the severity of pulmonary regurgitation. Am J Cardiol 2002;89:1438-41.

22 Hawkins JA, Thorne JK, Boucek MM, et al. Early and late results in pulmonary atresia and intact ventricular septum. J Thorac Cardiovasc Surg 1990; 100:492-7

23 Coles JG, Freedom RM, Lightfoot NE, et al. Long-term results in neonates with pulmonary atresia and intact ventricular septum. Ann Thorac Surg 1989;47:213-7.

24 Agnoletti G, Piechaud JF, Bonhoeffer P, et al. Perforation of the atretic pulmonary valve: long-term follow-up. J Am Coll Cardiol 2003;41:1399-403. 
25 Humpl T, Soderberg B, McCrindle BW, et al. Percutaneous balloon valvotomy in pulmonary atresia with intact ventricular septum: impact on patient care. Circulation 2003;108:826-32.

26 Justo RN, Nykanen DG, Williams WG, et al. Transcatheter perforation of the right ventricular outflow tract as initial therapy for pulmonary valve atresia and intact ventricular septum in the newborn. Cathet Cardiovasc Diagn 1997;40:408-13.

27 Gibbs JL, Blackburn ME, Uzun O, et al. Laser valvotomy with balloon valvoplasty for pulmonary atresia with intact ventricular septum: five years' experience. Heart 1997;77:225-8.

\section{IMAGES IN CARDIOLOGY}

\section{Double mycotic aneurysm of the descending thoracic aorta}

A 71 year old man was admitted with fever and chills. On admission his temperature was $39^{\circ} \mathrm{C}$; physical examination was normal except for a grade I/VI aortic systolic murmur. Blood cultures and urine samples grew Staphylococcus aureus. Transoesophageal echocardiography (TOE) showed a mildly calcified aortic valve without vegetations, and diffuse atheroma of the descending aorta. Despite appropriate antibiotic treatment the patient remained febrile, and developed clinical and radiological signs of pulmonary oedema. A subsequent TOE revealed the presence of two saccular aneurysms of the descending aorta, one just beneath the isthmus (panel A: AO, aorta), and the second one just above the diaphragm (panel B). These aneurysms arose from two localised ruptures in the atheromatous intima (arrows). The inferior one was responsible for a severe compression of the left atrium and right pulmonary veins, as shown by spectral and colour flow Doppler (panel C: LA, left atrium; LV, left ventricle; MA, mycotic aneurysm). Magnetic resonance imaging (spin echo $\mathrm{Tl}$ images) showed both mycotic aneurysms with a severely inflammatory shell and infiltration of the mediastinal fat, as well as the compressed left atrium (panel D: AA, ascending aorta; Pl.E, pleural effusion; RA, right atrium; RV, right ventricle; RPA, right pulmonary artery). The patient was considered too ill for surgery and died within a few days from multiorgan failure.

J-C Eicher

T Maurin

F Brunotte

J-E Wolf

jean-christophe.eicher@chu-dijon.fr
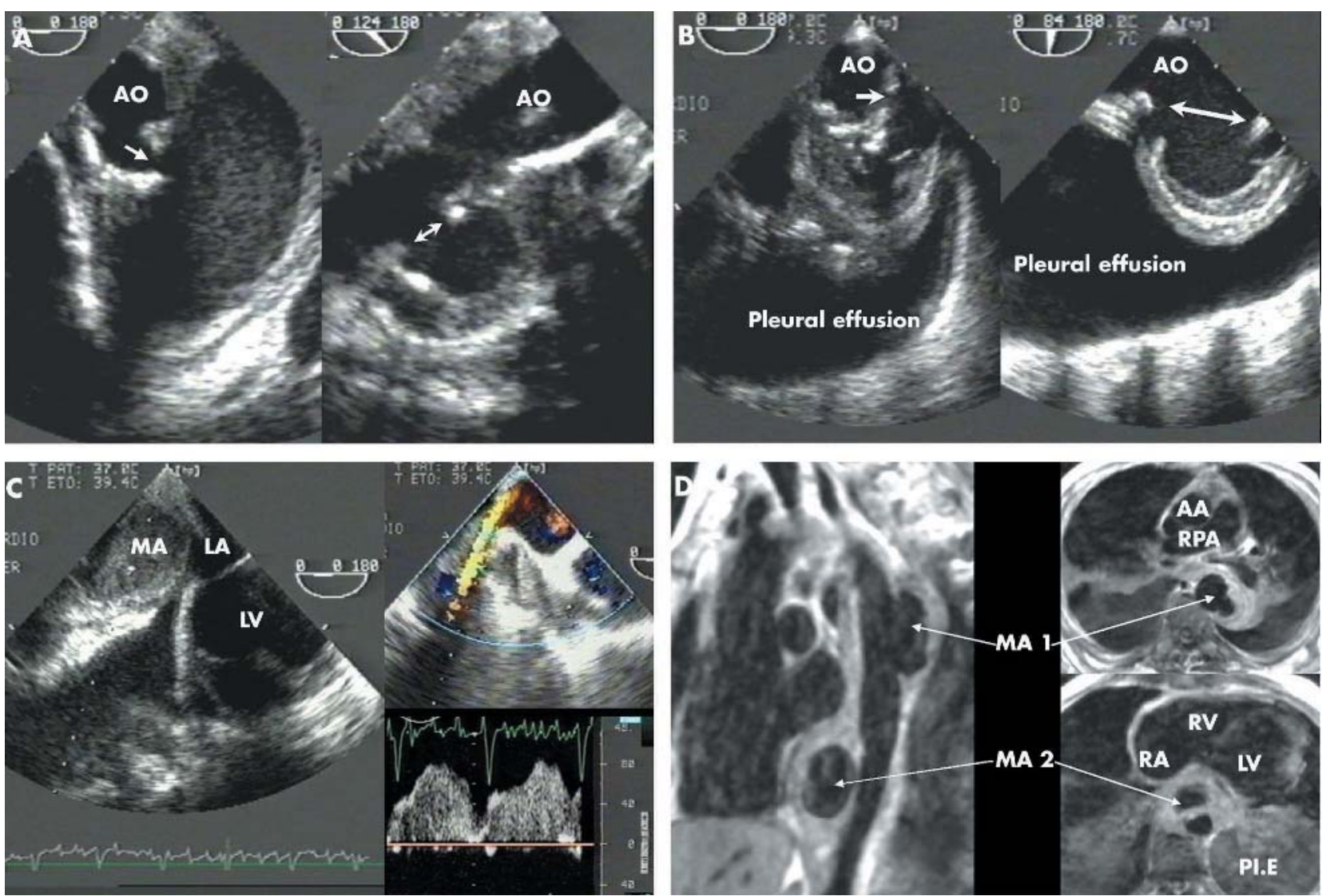